\title{
Las organizaciones de la sociedad civil en México: modalidades del trabajo y el empleo en la prestación de servicios de proximidad y/o relacionales
}

\section{Civil society organizations (cso) in Mexico: working modalities and employment in proximity and/or relational services}

\author{
Cristina Girardo* \\ Prudencio Mochi**
}

\begin{abstract}
This research addresses characteristics of the nonprofit sector taking as a reference the social economy theoretical framework and as analytic categories the definitions of activity, employment and work. According to this, we provide information about work and employment figures generated by proximity and relational services. We analyze a set of assets related to the generation of employment in this sector. This research offers knowledge in a scantly studied sector, and much less visualized neither from the academy nor the state sector.
\end{abstract}

Keywords: employment, activity, work, civil society organizations, proximity and/ or relational services, social economy.

\section{Resumen}

Este trabajo aborda las características del sector sin fines de lucro, tomando como referencia el marco teórico de la economía social y como categorías analíticas las definiciones en torno a actividad, trabajo y empleo. Se brinda información respecto a las modalidades de trabajo y empleo que se generan por la prestación de servicios de proximidad y/o relacionales. Se analizan un conjunto de activos que se relacionan con la generación del empleo en este sector. Se aporta con esta investigación al conocimiento del sector en un ámbito poco estudiado, tanto en la academia como en el sector público.

Palabras clave: empleo, actividad, trabajo, organizaciones de la sociedad civil, servicios de proximidad y/o relacionales, economía social. 


\section{Introducción}

En México, investigadores de distintas áreas han mostrado interés en el estudio del sector sin fines de lucro, que también ha sido llamado tercer sector, privado social, organizaciones de la sociedad civil, organizaciones no gubernamentales, economía solidaria, entre otros nombres. Sin embargo, todavía existen algunos aspectos asociados que permanecen casi inexplorados en los análisis de algunos académicos. Tal vez por tratarse de un ámbito percibido de manera muy confusa en el sistema de cuentas nacionales de México (SCNM) en cuanto al valor bruto de la producción (VBP), el valor agregado bruto (VAB), la remuneración de asalariados (RS), la formación bruta de capital fijo ( $\mathrm{FBCF}$ ) y el personal ocupado (PO) -como lo que es propio de la actividad mercantil lucrativa-, no encontramos, por ejemplo, investigaciones que abordan el empleo o las oportunidades de trabajo que se generan en este ámbito. Hablamos de un sector en crecimiento durante estos últimos años, con una notable participación de ciudadanos y una pujante contribución al bienestar de la gente.

En este trabajo pretendemos dar cuenta de las modalidades de trabajo y empleo que se generan por la prestación de servicios de proximidad y/o relacionales, brindados por el sector sin fines de lucro en México. Algunos interrogantes que se plantean son: ¿cuáles son las peculiaridades que distinguen a las organizaciones que forman parte de este sector?, ¿en qué esfera de la economía podemos situar sus actividades?, ¿qué clase de bienes y servicios son producidos y distribuidos según las reglas propias de algunas de las organizaciones sin fines de lucro?, ¿a qué población atienden?, ¿¿cuáles son las modalidades del trabajo y del empleo en estas organizaciones? Para responderlos, nos detenemos primero a analizar algunas categorías analíticas que servirán como referente y marco teórico al análisis del trabajo en el sector. Luego damos cuenta de algunos resultados de la investigación titulada "El empleo y sus modalidades en las organizaciones de la sociedad civil", financiada por el Conacyt (2007-2009). El objetivo de esa investigación consistió en aportar un análisis cuantitativo y cualitativo de los recursos humanos que laboran en las organizaciones de la sociedad civil (OSC) en México, con referencia especial a su evolución reciente y a las expectativas de crecimiento de las propias entidades. Para ello se diseñó una encuesta mediante la aplicación de un cuestionario semiestructurado entre funcionarios (directivos) y personal de las osc. Diseńamos una muestra proporcional y estratificada por cada estado del país. Para aplicar la entrevista dentro de cada estrato (el estado) no consideramos el tipo de organización (fundaciones, asociaciones civiles, instituciones de asistencia privada u otras), sino que se aplicó de manera 
aleatoria y sólo se sujetó a que los convocados contestaran los correos electrónicos y/o las llamadas telefónicas.

El ámbito contemplado fue nacional; el periodo de levantamiento de la encuesta se ubicó entre el 26 de junio y el 14 de agosto del 2007 en casi todos los estados de la República Mexicana. ${ }^{1}$

La mayor parte de los entrevistados respondió la encuesta por correo electrónico y, cuando así lo prefirieron sólo algunos, por teléfono. El marco muestral quedó constituido mediante el Directorio de Inscripción al Registro Federal de Organizaciones de la Sociedad Civil para obtener la clave única, conocida como Cluni. El registro está a cargo del Instituto Nacional de Desarrollo Social (Indesol), del que formaban parte al momento del levantamiento de la encuesta 6,826 organizaciones. En total, trabajamos con 1,078 casos como muestra.

\section{Características del sector sin fines de lucro}

La creciente importancia del sector no lucrativo en México nos obliga a acotar con algunos criterios delimitadores lo que entendemos por este sector y qué tipo de organizaciones incluye. El conjunto de ellas constituye un universo heterogéneo y diverso dentro del cual se puede distinguir una tipología variada en lo político, social e ideológico. Por esta diversidad es difícil encontrar un contenido normativo común (Mochi, 2003). En realidad, la amplia heterogeneidad de los actores que participan y trabajan en este sector, así como la ausencia de normativas nacionales que uniformen, tipifiquen y regularicen esta categoría, impiden la formalización de una definición unívoca.

Una breve revisión de las ciencias sociales en general y de la economía en particular (Ackeman, 1996) pone de manifiesto la dificultad que entraña, desde el marco teórico conceptual (Donatti, 1996; Girardo, 2003, 2005, 2007; Olvera, 1999), el análisis de un sector asentado en el principio de no lucrar. Sin embargo y a pesar de estos límites, se han realizado magníficas aproximaciones teóricas (Weisbord, 1991) y empíricas (Clotfeler, 1992) a este sector institucional, que tiene una notable y creciente presencia en ciertas actividades económicas, sociales y culturales, además de efectos significativos sobre la eficiencia y la equidad.

Una característica peculiar de las osc es su pertenencia a una dimensión institucional poco común: lo público no estatal (Bresser y Cunill, 1998). Estas organizaciones actúan como sujetos en nombre del bien público. Son expresión de la ciudadanía activa. La innovación que más

\footnotetext{
${ }^{1}$ Campeche, Colima, Durango, Estado de México, Guanajuato, Guerrero, Hidalgo, Sinaloa, Tabasco, Tlaxcala, Aguascalientes, Chiapas, Chihuahua, Coahuila, Distrito Federal, Jalisco, Michoacán, Morelos, Oaxaca, Querétaro y Sonora.
} 
destaca es que introducen, dan forma (política y organizativa) a un cambio en las estrategias de la ciudadanía. Va más allá de la tradicional dicotomía entre lo público y lo privado, supera la coincidencia entro lo público y el Estado, a la vez que redefine esas nociones. Para distinguir lo público de lo estatal y de lo corporativo, entendido como algo que es de todos y para todos, se lo opone en este sentido tanto a lo privado (volcado al lucro o al consumo) como a lo corporativo (orientado a la defensa política de intereses sectoriales: partidos políticos y sindicatos) (Girardo, 2003). Las instituciones que componen este sector son las organizaciones no gubernamentales (ONG), las asociaciones, las organizaciones de base o de apoyo, de defensa de los derechos, las organizaciones ciudadanas; instituciones de asistencia privada, asociaciones de vecinos, fundaciones; organizaciones de tipo cultural, de matriz religiosa, deportivas y de esparcimiento; urbanas territoriales, de comunidades indígenas, de estudios y análisis políticos; entre muchas más. Se las denomina tercer sector, economía solidaria, organizaciones de la sociedad civil, no gubernamentales, instituciones de asistencia privada, sin fines de lucro, de promoción social y desarrollo, organizaciones civiles... Y si bien no todas estas expresiones significan lo mismo, sí expresan la necesidad de acotar una realidad específica: representar a organizaciones que buscan el bienestar de las personas, por medio de sus acciones o de su injerencia en la construcción de la agenda pública.

Una segunda peculiaridad que las distingue respecto de los otros sectores - Estado y mercado-, es que las organizaciones empeñadas en erogar servicios a la comunidad satisfacen necesidades asociadas con valores de uso no monetario, y en general se trata de bienes relacionales intangibles (Caselli, 1998; Donatti, 1996; Fiorentini, 1997) cuyas necesidades el Estado no siempre es capaz de atender, o mejor dicho, de comprender dadas sus características novedosas, ajenas a la dinámica de las necesidades comunes (más universales) que generalmente el Estado atiende mediante las políticas del Estado de bienestar. Las osc colocaron en la agenda -mucho antes que el Estado social- políticas públicas para combatir problemas ligados con la atención a la violencia intrafamilar, a los temas del consumo de drogas, de la discapacidad o de la migración, entre muchos otros más.

Una tercera característica distintiva son los recursos de que disponen estas organizaciones, tanto financieros como humanos. Se trata de las donaciones (provenientes de la cooperación internacional, nacional y de ciudadanos), así como de los recursos humanos voluntarios, sin paralelo en la empresa privada y en la administración pública. Esta distinción les permite, al menos en teoría, que puedan disponer de recursos ilimitados, siempre que estén en condiciones de saber y poder atraer suficientes donativos o trabajo voluntario (Fiorentini, 1997). Asimismo, a estos beneficios se suma el hecho de que últimamente en México muchas organiza- 
ciones han venido experimentando nuevas formas de autosustentabilidad, merced a la prestación de servicios (venta de productos, tiendas solidarias, comercio justo), sobre todo, muchas de ellas están recibiendo financiamiento del gobierno como una forma de corresponsabilidad para gestionar servicios. Esto aporta trabajo remunerado para la consecución de metas que nutren el bien general o el interés común.

Pero, fundamentalmente, una peculiaridad que las distingue de los demás sectores son los ideales y la motivación de las personas que integran tales organizaciones, pues constituyen prerrequisitos indispensables para ingresar al sector. Sin embargo, estos ideales ya no son suficientes para garantizar de manera eficiente el éxito y la continuidad de las osc. La experiencia demuestra que las organizaciones necesitan contar con el valor agregado en términos de competencias concretas, transparencia y rendición de cuentas que les permitan legitimar sus acciones frente a otros ciudadanos y a las instituciones.

Las peculiaridades descritas no pretenden eximir del análisis una visión más integral de estas organizaciones, en la que es necesario señalar aspectos que contradicen la tendencia de identificar a las osC sólo como entidades honestas, justas y transparentes. Por una parte, podemos señalar que existen organizaciones racistas, antidemocráticas o fundamentalistas, así como otras que atentan contra los derechos de los grupos sociales y políticos, lo que redunda en un capital social negativo. También las debilidades que enfrentan estas organizaciones para su funcionamiento las condicionan para alcanzar metas de eficiencia, continuidad a largo plazo, transparencia, entre otras. Entre estas debilidades se cuentan: la incertidumbre sobre la capacidad de financiamiento que condiciona la supervivencia de la organización y la realización de muchos proyectos, con una visión de más largo alcance; el protagonismo otorgado en muchas ocasiones a los líderes fundadores, a quienes se les conceden atributos que minan la democracia dentro de las mismas organizaciones; la poca o nula inversión en profesionalización de sus miembros; la incorporación de procesos de rendición de cuentas, entre muchos más. En síntesis, de este universo tan heterogéneo surge un sector que debe ser entendido en su segmentación, heterogeneidad y diversidad en múltiples aspectos.

\section{La noción de potencialidad como conjunto de activos}

Esta noción de potencialidad como conjunto de activos (Guadarrama y Girardo, 2008) incorpora el principio de que las organizaciones pueden convertirse en generadoras de empleo a medida que satisfagan necesidades sociales. La noción de activo implica que toda organización existe en la medida de unos fines determinados; es relativamente permanente, reco- 
noce una división de tareas, y tiene reglas de funcionamiento (Selznick, 1996), lo que se interpreta en el sentido propuesto por Cabin (1999) al señalar que las organizaciones existen cuando cuentan con algún tipo de estructura, recursos y un sistema de gestión. El conjunto de activos articula las posibilidades y limitaciones de las organizaciones para ser sustentables y eficientes en la consecución de sus objetivos; también su importancia en la generación de empleo.

En una primera exploración (Guadarrama y Girardo, 2008) se consideraron como activos en la generación de empleo los siguientes:

- La modalidad de adscripción del personal voluntario o remunerado.

- El tipo de contratación del personal: permanente, por tiempo determinado, por trabajo provisional, por cuenta propia, voluntario, remunerado.

- La formalidad o informalidad del empleo.

- El perfil profesional del trabajador.

- El tipo de actividad profesional que desarrolla.

- La capacitación, el entrenamiento del personal y los servicios que brindan.

- Los soportes financieros que limitan o incentivan distintas modalidades de empleo.

\section{La economía solidaria como marco de referencia}

En muchos países, sobre todo europeos -y ahora cada vez más en México y en otros de América Latina-, se habla de economía solidaria para englobar las actividades llevadas a cabo por este sector, cuyo objetivo es la satisfacción de un interés general o mutuo, de contribución al bien común o de respuesta a demandas sociales expresadas por ciertas categorías de la población. Este concepto - por cierto, mucho más amplio que el término no lucrativo- abarca una gran cantidad de organizaciones en forma de cooperativas, mutuales, empresas sociales, cuya frontera no está delimitada por el hecho de ser clasificadas con o sin fines de lucro, sino porque privilegian la constitución de un patrimonio colectivo en relación con el retorno sobre la inversión individual. En este sentido, el término tercer sector usado por la cultura anglosajona (Salomón y Anheimer, 1997, 1998) toma como referencia el criterio de no redistribución como eje de clasificación de las organizaciones que entran en este archipiélago, excluyendo cooperativas, mutualidades y muchas otras experiencias vinculadas con economías comunitarias. ${ }^{2}$ Para nuestro trabajo y al considerar el valor

\footnotetext{
${ }^{2}$ En este sentido conviene consultar a Verduzco (2003: 112), que describe todas las formas de organización que no entran en los trabajos de Salomon y Anheimer, quienes además toman como referencia una definición estructural operativa para clasificar las organizaciones que entran en esta definición.
} 
económico y social de los servicios que producen las osc en general, se puede considerar que las mismas llegan a ser ubicadas en el sector de actividad económica perteneciente a la economía solidaria (Guadarrama y Girardo, 2008). Aunque cabe aclarar que no hemos considerado en esta primera fase de la investigación el estudio de las cooperativas, ni de las mutuales o de las empresas sociales y comunitarias en sentido amplio. Tampoco hemos considerado otras actividades que se incluyen en la economía solidaria y que son, por ejemplo, el comercio justo, algunas actividades de microfinanciamiento, la promoción y el desarrollo de energías renovables, las actividades de promoción y aplicación del software libre, las ferias comunitarias, la agricultura biológica, el ecoturismo, entre otras.

Los servicios que las organizaciones examinadas en este trabajo realizan se orientan principalmente a la prestación de servicios que procuran el bienestar del otro (atención a ancianos, niños de la calle, enfermos, discapacitados; profesionalización de ciertos sectores, empoderamiento y formación de liderazgos, entre algunos de los muchos identificados). Analizamos esto bajo el concepto de servicios de proximidad y/o relacionales, descritos a continuación.

\section{Los servicios de proximidad o de relacionalidad}

Si bien los servicios denominados de proximidad y/o relacionalidad (Laville, 2004; Donatti, 1996) no constituyen aún un sector identificado y constituido en México, se puede apreciar que la combinación de recursos y los servicios que proveen las organizaciones civiles se pueden asociar con las características que asume ese sector en otros países, particularmente europeos, ${ }^{3}$ y que el concepto comienza a escucharse en el país, sobre todo en el ámbito de los estudios y programas de economía solidaria.

Estos servicios de proximidad ${ }^{4}$ pueden definirse como "aquellos que a partir de un impulso de reciprocidad, operan una construcción conjunta de la oferta y la demanda cuya consolidación pasa por la combinación con los principios del mercado y la redistribución" (Lavillle, 2004: 43).

Este tipo-ideal de servicios se caracteriza por crearse y consolidarse entre diferentes tipos de recursos: mercantiles, no mercantiles y no monetarios.

${ }^{3}$ Éstos se han reconocido como componentes de nuevos servicios que las políticas empezaron a sostener en el llamado Libro Blanco, documento de reflexión de la Comunidad Europea para el siglo xxi donde se anunciaba "una cifra de tres millones de nuevos empleos" que podían distribuirse "igualmente entre los servicios de proximidad, la mejora del marco de vida y la protección del medio ambiente" (Comisión Europea, 1993: 13).

${ }^{4}$ La Comisión Europea en el año 1995 (Les initiatives locales de développement et d'emploi), en el Libro Blanco, individualizó cuatro grandes sectores para focalizar este tipo de servicios: los servicios de la vida cotidiana, los servicios para mejorar las condiciones de vida, los servicios culturales y recreativos y los servicios para el ambiente. 
Asimismo, se les concibe como una inclinación de reciprocidad que permite una construcción conjunta de la oferta y la demanda por medio de espacios públicos de proximidad. Esta categoría de servicios de proximidad no se restringe a servicios sociales de tipo asistencial; el rubro se puede definir como servicios que responden a necesidades personales o colectivas, basados en una relación de proximidad objetiva o subjetiva, referidos al espacio o tiempo, así como a la subjetividad de los actores. La proximidad puede adoptar forma de colonia o de municipio, ya que los servicios se brindan en lugares restringidos y responden de manera oportuna a la demanda de los usuarios, aunque no se confunde con la colonia y/o el municipio, sino que la proximidad se define por el hecho de que es sentida, experimentada e interiorizada por los actores (dimensión relacional del servicio, marcada por la relación directa entre el proveedor del servicio y quien lo recibe), y no por referencia al territorio (Laville, 2004: 39).

Encontramos la innovación en cuanto que estos servicios de proximidad se apoyan en el principio de reciprocidad, que es un principio de comportamiento económico distinto al del mercado y la redistribución. El recurso al mercado y la distribución en el funcionamiento de las instituciones microeconómicas aumentan el impulso de reciprocidad. Se refiere así una articulación permanente entre la reciprocidad, el mercado y la redistribución. Este híbrido confiere márgenes de maniobra suplementarios, lo que mejora la accesibilidad a los servicios y reduce los costos tanto para el usuario como para las finanzas públicas. En este sentido, el principio de reciprocidad guía las conductas para elaborar servicios sobre un modo igualitario y según un proceso de interacción entre personas implicadas de modo voluntario. Como las denomina el mismo Laville, también pueden llamarse servicios solidarios. El impulso de reciprocidad "genera la construcción conjunta de la oferta y la demanda" [...]. Los servicios de proximidad se fundan en las relaciones y los intercambios simbólicos que se tejen en la trama diaria de la vida local, los anhelos, valores y deseos de quienes son sus usuarios (Laville, 2004: 40; cursivas en el original).

La confianza es un valor imprescindible y decisivo en la prestación de estos servicios debido a que penetran en la intimidad de los usuarios (la confianza tanto en los sistemas expertos como hacia las personas [Giddens, 1994, citado por Laville, 2004: 41]). En especial, este segundo tipo de confianza basada en el compromiso cara a cara, en el encuentro entre personas, así como la copresencia. No se trata sólo de la expresión de una demanda como en los servicios sociales tradicionales, en los cuales ésta adoptó el hábito de moldearse sobre la oferta disponible. Se trata más bien de la formación de una "confianza interpersonal que se vuelve posible por el desarrollo de sociabilidades primarias fundadas en la búsqueda de un vivir mejor colectivo" (Eme, 1993: 69). Esto se expresa en espacios 
públicos de proximidad (Eme, 1993) constituidos por usuarios, voluntarios o profesionales. Una esfera pública que supera lo individual y privado, una esfera de la palabra y de la acción en común que se distingue por su apertura de la esfera privada de la familia (Chanial, 1992, citado por Laville: 2004). No se trata, como se esfuerzan en describirlo los autores citados en este párrafo respecto de los servicios domésticos, ni de estimular un retorno a la familia (lugar de solidaridades naturales), tampoco de servicios procedentes de simples relaciones de vecindario (solidaridades espontáneas); si bien es cierto que en muchas ocasiones los servicios de proximidad se apoyan en recursos domésticos (atención a la tercera edad, a niños discapacitados, etc.), para reforzarlos y no para aislarlos, ratificando el encierro de las familias. En este sentido se definen proyectos de intervención a fin de aliviar las tensiones y proteger el equilibrio familiar mediante intervenciones profesionales. El usuario es determinante para constituir la oferta, ya sea por iniciativa propia o porque forma parte de los mismos trabajadores sociales, o aun por la intervención de profesionales que llegan a percibir las demandas insatisfechas al quedar involucrados de manera directa en la producción de servicios.

\section{Actividad, trabajo, empleo como categorías analíticas}

La utilidad de ciertas categorías teóricas y estadísticas para analizar tanto las actividades que se realizan, el trabajo que se desempeña, así como el empleo que generan en el sector no lucrativo, representa un aspecto esencial para analizar la realidad del mercado laboral en este sector. El principal problema planteado es, sin duda, la medición de estas categorías, dado que para estudiar un mismo fenómeno se emplean diferentes conceptos y/o métodos de recolección de datos. Además se suma la inconsistencia -como veremos a lo largo de todo este trabajo- que encontramos en un sector tan disperso y poco sistematizado como éste, conformado por instituciones de asistencia privada, fundaciones, asociaciones civiles, que hemos denominado de manera genérica organizaciones de la sociedad civil sólo para fines de este estudio. Tendremos pues que considerar otras dimensiones teóricas que demuestren diferentes facetas relacionadas con las actividades, el trabajo y el empleo en el sector, como son, por ejemplo, las motivaciones 5 que impulsan a las personas a trabajar en este tipo de organizaciones, sus principios reguladores, el uso del tiempo, la oportunidad de realizar trabajos más compensatorios por su innovación y el aprendizaje que desarrollan, entre otras.

\footnotetext{
${ }^{5}$ Estos aspectos también se indagan en nuestra investigación financiada por Conacyt, y llevados a cabo a través de un estudio cualitativo que estamos realizando ahora.
} 
Si nos basamos en los instrumentos tradicionales para medir el empleo en las osc, encontramos que existen muchas imperfecciones en los instrumentos tradicionales; por ejemplo, en las cuentas nacionales, donde no aparece una distinción de este tipo de trabajadores. Por otra parte, las definiciones tienen un cierto grado de ambigüedad. Estas imperfecciones se ven retroalimentadas por tratarse de un sector -sobre todo en Méxicoinvisible o poco reconocido como un sector autónomo y sistematizado desde sus propias características.

Al tomar como referencia la distinción conceptual entre actividad, trabajo y empleo (Neffa, 2005: 12-15) se establece que la actividad es una noción muy amplia que expresa todo el dinamismo de la naturaleza humana; el trabajo tan sólo es una de ellas, al lado de las actividades desarrolladas en las esferas doméstica, educativa, cultural, deportiva, sindical, política, etc. El trabajo es una actividad efectuada por las personas, orientada hacia una meta: la producción de un bien, o la prestación de un servicio, lo que da lugar a una realidad objetiva, exterior e independiente del sujeto, y socialmente útil para la satisfacción de una necesidad. El trabajo involucra a todo el ser humano, y no únicamente en sus dimensiones fisiológicas y biológicas, porque al mismo tiempo moviliza las dimensiones psíquicas y mentales. Por regla general, existe una gran diferencia entre el trabajo, tal como lo prescriben quienes tienen la responsabilidad de su concepción, y la manera como se ejecuta; moviliza no sólo el esfuerzo, la formación profesional y la experiencia acumulada, sino además la creatividad, el involucramiento y la capacidad para resolver problemas y enfrentar los incidentes cotidianos en las empresas y organizaciones.

Ahora bien, cuando el trabajo así definido se ejerce para obtener ingresos, en calidad de asalariado, de empleador o aun actuando por cuenta propia, estamos en presencia del empleo, que puede desarrollarse en el ámbito mercantil o en actividades sin fines de lucro, y llevarse a cabo sin relación de dependencia o como asalariado.

De esto se deduce que todas las personas que emprenden una actividad laboral, por eso mismo están ocupadas, lo cual no significa que tengan un empleo asalariado. Por desgracia, no siempre es posible traducir estas definiciones conceptuales (que tienen hoy día gran relevancia) en las categorías estadísticas operatorias.

Otra distinción importante -toda vez que se afronta el análisis del trabajo realizado por un intercambio económico-consiste en considerar la imposibilidad de inscribirse en la categoría fundamental de trabajo asalariado por tiempo indeterminado. Si bien es cierto que el tipo de trabajo remunerado que domina los escenarios actuales se caracteriza por la flexibilidad, las diferencias de sus modalidades de prestaciones son tantas que sugieren la hipó- 
tesis de que la nueva realidad en el mercado laboral está compuesta por muchas figuras, con habitus mentales distintos y contrapuestos, y con variadas formas de contratos y maneras de ejercer el trabajo.

\section{Activos en la generación de empleo en las osc de México}

\subsection{La modalidad de adscripción del personal: voluntario y remunerado}

Las OSC no representan en sí mismas un sector cuyo número de personas empleadas devenga importante. Si tomamos en cuenta que la PEA en México está conformada por 45’460,003 personas, según la Encuesta Nacional de Ocupación y Empleo (ENOE), con cálculos proporcionales propios y de acuerdo con el número de organizaciones que contestaron la encuesta de nuestra investigación $(1,078)$, quienes dieron cuenta de 11,432 personas laborando de manera remunerada dentro de las organizaciones; resultan en total sólo 11 personas por organización. Podemos intuir (con un total de 6,826 organizaciones de nuestro directorio) que sólo un porcentaje menor estaría involucrado en este tipo de organizaciones, con casi $0.14 \%$ de la PEA. Sin embargo, es necesario recordar la posibilidad de ampliación de este universo si se aplicara un instrumento legislativo que nos permitiese individualizar el sector en su conjunto con una categoría de trabajadores; y si además en nuestro universo se hubiera considerado al sector de actividades vinculadas con cooperativas, mutuales, empresas sociales, entre otras.

Las osc cuentan con recursos humanos asalariados y con otras formas de contratación (pertenecientes a la esfera mercantil, pero también bajo un esquema particular, dada su condición de no lucro); además, integran voluntarios (trabajo libre en la esfera no mercantil representado por el trabajo voluntario y el trabajo militante, donde no se pagan salarios ni existe relación de dependencia). En la gráfica I se observa que en las organizaciones involucradas, $59.5 \%$ de los trabajadores (denominados en la encuesta de manera general como "remunerados") labora de tiempo completo, mientras que $40.5 \%$ trabaja de forma parcial; y para el trabajo voluntario, $22.3 \%$ está de tiempo completo y $77.7 \%$ asiste de manera parcial. Estos datos evidencian la manera esporádica de relacionarse los voluntarios con el trabajo realizado dentro de las organizaciones. En muchos casos esto ocasiona tensiones entre los trabajadores remunerados de tiempo completo y los voluntarios, ya que al involucrarse por poco tiempo los voluntarios - a los ojos de los otros trabajadores- son vistos como poco efectivos para realizar sus actividades. 


\section{Gráfica I \\ Porcentaje de voluntarios y remunerados según el tipo de jornada}

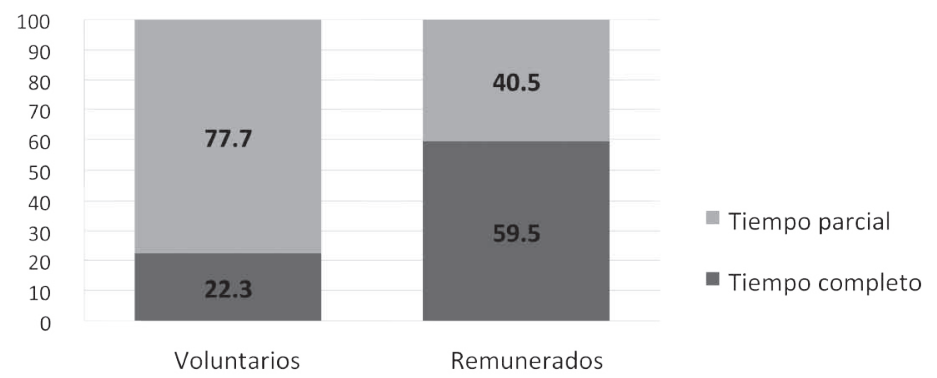

Fuente: Elaboración propia, 2008. Proyecto Conacyt.

\subsection{Modalidades de contratación del personal remunerado}

En la gráfica II se puede discernir el tipo de contrato con el que laboran los "remunerados", quienes trabajan 63\% de tiempo completo e indefinido; $10.1 \%$ labora de tiempo completo pero sólo por temporadas (probablemente el tiempo vinculado a un proyecto dado); $16.3 \%$ va de manera parcial y por un lapso indefinido (es el trabajo de medio tiempo); y $10.6 \%$ firma contrato parcial y temporal (se refiere a consultorías esporádicas, o a trabajo por honorarios).

\section{Gráfica II}

Porcentaje de voluntarios y remunerados según el tipo de contrato

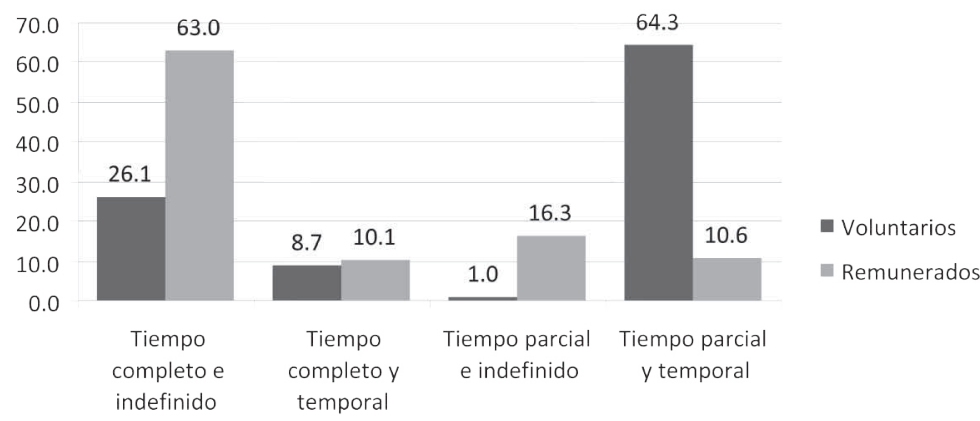

Fuente: Elaboración propia, 2008. Proyecto Conacyt. 


\subsection{La formalidad o informalidad del empleo: los trabajos atípicos}

Los trabajos realizados en las osc reciben poca remuneración ${ }^{6}$ (cuadro 1), aunque es menester señalar el bajo porcentaje de respuestas a esta pregunta. Tampoco se pudo constatar la cantidad de pagos generados en otros esquemas de contratación (por honorarios, por proyectos a término, por consultorías y demás), que aunque es probable que estén mejor pagados, son mucho menos estables.

Cuadro 1

Salarios

\begin{tabular}{lccc}
\hline & Directivos & Profesionales & Administrativos \\
\hline Salario bruto promedio & $\$ 9,812.00$ & $\$ 7,110.00$ & $\$ 4,666.00$ \\
\hline
\end{tabular}

Fuente: Elaboración propia 2008. Proyecto Conacyt.

En la mayoría de los casos no existe un contrato de trabajo escrito; más bien, se trata de empleos que no están regulados exclusivamente bajo principios utilitarios de la racionalidad técnico-instrumental. Desde el punto de vista de la regulación del trabajo, suelen confundirse los derechos de los trabajadores con los del cliente (o usuario/beneficiario de los servicios). La interacción es entre actores clave del proceso productivo; de este modo, por ejemplo, el usuario y/o beneficiario también interviene en el proceso, pues al ejercer control sobre los precios, la calidad del producto, así como la construcción de conflicto o de consenso continuamente están presentes. En la construcción sociodemográfica del mercado laboral, el usuario o beneficiario debe ser contemplado porque influye en la oferta y demanda de los trabajadores y sus características. También incide en la regulación del trabajo porque los derechos y obligaciones del usuario o beneficiario deben ser considerados junto con los derechos y obligaciones de las organizaciones, que en este caso operan con carácter patronal.

En la gráfica III se describe el tipo de prestaciones sociales de los trabajadores. Encontramos que $86.2 \%$ no tiene prestaciones; sólo $5.1 \%$

${ }^{6} \mathrm{Si}$ tomamos alguna referencia y comparamos los ingresos en la administración pública (federal o estatal), teniendo en cuenta que los ingresos varían según la dependencia gubernamental de la cual se trate, observamos una fuerte diferencia con los salarios del sector no lucrativo. Las secretarías económicas tienen mejores ingresos - pensando en las mismas categorías del tabulador-, por ejemplo Hacienda y Economía son categoría "A" y la del Trabajo o Reforma Agraria son nivel "C". En muchas ocasiones suelen manejarse compensaciones y sobresueldos que no siempre se conocen los montos. Un director general gana alrededor de 100,000 pesos mensuales, un director de área unos 70,000 pesos; los subdirectores entre 45,000 y 50,000 pesos y los jefes de departamento entre 15,000 y 18,000 pesos. 


\section{Gráfica III \\ Qué prestaciones tienen los trabajadores asalariados \\ (porcentajes)}

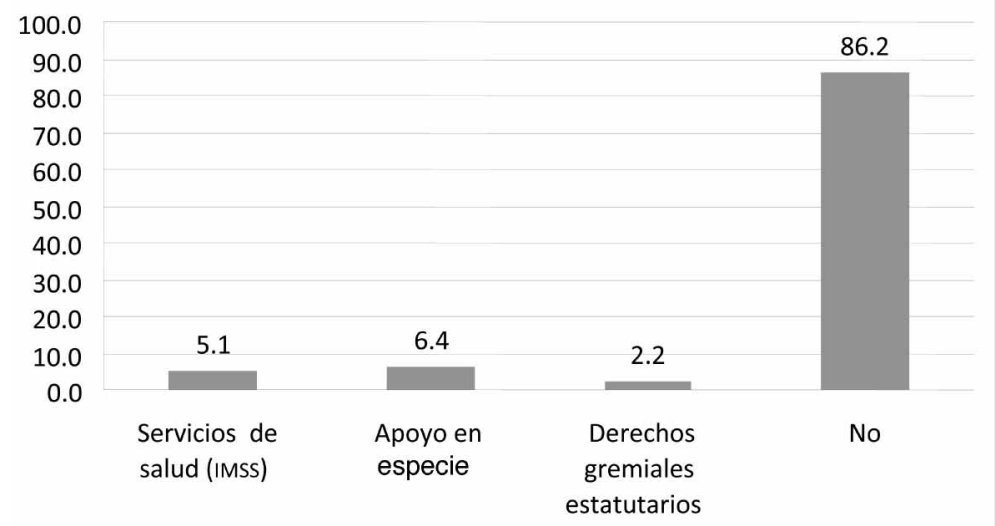

Fuente: Elaboración propia 2008. Proyecto Conacyt.

cuenta con servicios de salud del IMss, y $6.4 \%$ percibe prestaciones en especie (si bien no se especifica cuáles) y 2.2\% sólo recibe las que otorgan los derechos gremiales estatutarios. Estos datos evidencian una falta casi total de prestaciones sociales, hecho que coloca a este sector de trabajadores en una situación precaria. Los demás trabajadores remunerados, cuando no se procuran ellos mismos su propia afiliación a algún servicio privado de salud, quedan totalmente desprovistos de esos beneficios.

\subsection{El tipo de actividad profesional y el entrenamiento del personal}

Trabajar en una osc significa no sólo una elección de trabajo o una vía para hacer carrera; es sobre todo una elección de estar en el mundo diversamente, de compartir objetivos comunes. Claro que se puede estar en el mundo de manera responsable sin necesidad de colaborar en las osc; pero se requieren ideales y motivación específicos para trabajar en ese sector. Se habla de una cierta identidad de los trabajadores sociales, aunque no es rara su dificultad para responder las preguntas "¿qué trabajo haces?” o “¿qué profesión tienes?” Sus respuestas varían: "Trabajador social”, "Trabajo en una organización no gubernamental de psicólogo", "Soy educador", "Animador"... Ellos manifiestan su dificultad para definir lo que 
significa ser un agente de desarrollo social.7 Se trata de una cuestión que patentiza no sólo algunos problemas lingüísticos, sino más bien un defecto en la definición del objeto de trabajo, los conocimientos, las habilidades, las competencias transversales que ayudan a caracterizar la acción profesional. Esta indeterminación conduce a los actores hacia la indefinición propia debido a tantas ambigüedades del sector.

Los ideales y motivaciones ya no son suficientes para ejecutar servicios de manera óptima. La experiencia demuestra que las organizaciones necesitan un valor agregado en términos de competencias concretas. Cada vez más se vuelve necesario el desarrollo de una profesionalidad definida muy específica, rica en capacidad operativa, concreta y congruente con las necesidades de los servicios que se demandan. Para abordar el tema del trabajo dentro de las osc, la formación y capacitación aparecen como herramientas con potencial, aportándoles elementos atendibles para que las personas que participan en el sector, así como las propias organizaciones, desarrollen capacidades idóneas, a fin de que logren desenvolverse en las actividades que realizan cotidianamente, además de generar los flujos eficientes de distribución del conocimiento en el territorio.

La individualización de estos profesionales que actúan en lo social se torna cada vez más preponderante, ya que inciden en su trabajo no sólo con el aumento de ocupación, sino también en la calidad de vida de las personas, en la lucha contra la pobreza, rediseñando e imponiendo nuevas condiciones de crecimiento y participación sociales.

En nuestro trabajo de investigación identificamos que los profesionales del sector, aunque proceden de profesiones más bien tradicionales (gráfica IV), responden a una serie de necesidades nuevas impuestas, por una parte, por todas las transformaciones del Estado social, descritas en párrafos anteriores, que condicionan y modifican el trabajo social y a los trabajadores del sector. Los servicios se encuentran atiborrados de exigencias por parte del ciudadano. Es en este contexto donde el trabajo en el ámbito del desarrollo social se coloca frente a nuevos e ingentes desafíos. La realidad social se aprehende en su complejidad y la creatividad del profesional se pone al servicio de la construcción de propuestas inéditas de intervención profesional. Por otra parte, estos trabajadores se caracterizan por un tipo de profesionalidad que denominamos emergente y que en otros textos se definen como agentes de desarrollo social, ligados al compromiso generado por sus propias motivaciones sectoriales.

El agente de desarrollo social es un operador, dotado de competencias para promover el desarrollo de la colectividad, para actuar en el campo

\footnotetext{
7 Véase el informe de investigación para el diseño de una maestría en agentes de desarrollo social, México, Indesol, 2005, coordinado por Cristina Girardo para El Colegio Mexiquense, A.C.
} 


\section{Gráfica IV \\ Perfiles profesionales de los agentes que trabajan para las OSC}

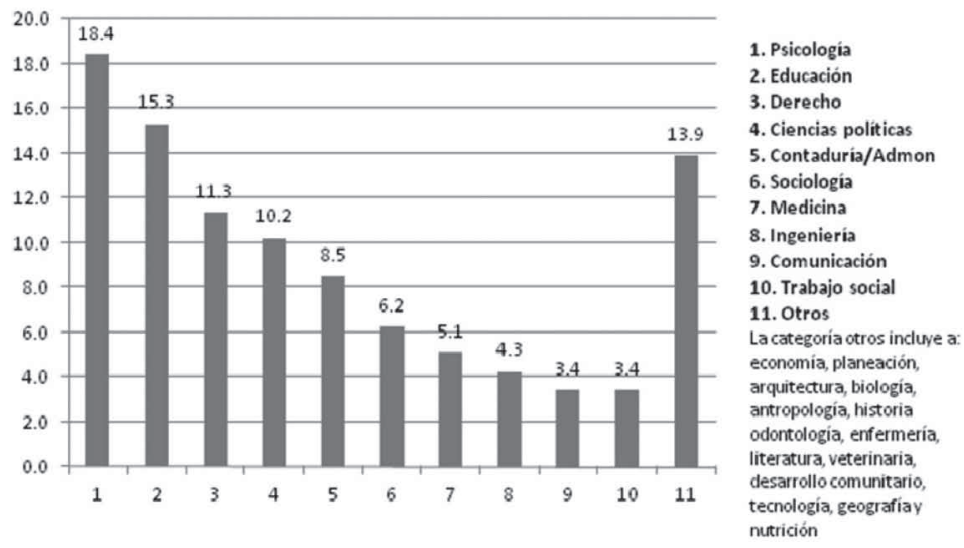

Fuente: Elaboración propia, Proyecto Propuesta Maestría en Agentes de Desarrollo Social, Indesol, 2005.

de la administración pública, en las osc, en universidades, lo mismo que en empresas del sector privado suscritas bajo el principio de la corresponsabilidad social empresarial.

Debe ser capaz de promover la construcción de vínculos entre sujetos e iniciativas, de analizar el contexto socioeconómico y cultural; de gestionar y evaluar proyectos que favorecen procesos de cooperación nacional e internacional; de diseñar políticas públicas y de utilizar los instrumentos normativos vigentes. Es, también, un facilitador del desarrollo con equidad de género.

De acuerdo con las competencias ${ }^{8}$ identificadas para desarrollar su labor, este profesional debe contar con nuevas destrezas relacionales,

\footnotetext{
${ }^{8}$ En la investigación se han identificado toda una serie de competencias que mencionamos de manera general: realizar análisis estadísticos, económico-sociales y culturales del territorio; elaboración y gestión de estrategias de desarrollo social en los ámbitos nacional, estatal y, sobre todo, local, con equidad de género; promover procesos de concertación entre las instituciones del sector público, la sociedad civil, las empresas privadas, promoviendo sus correspondientes alianzas estratégicas, a fin de sostener y orientar la producción de bienes públicos; analizar y evaluar las políticas públicas y las inversiones de presupuesto en materia de desarrollo social; utilizar los instrumentos normativos que regulan el sector del desarrollo social. Promover programas que generen igualdad de oportunidades; aplicar los principios del desarrollo sustentable y sostenible, así como de impacto ambiental desde la perspectiva de los derechos humanos. Tales competencias se integrarán con otras habilidades técnicas y aptitudes relacionales y motivacionales transversales, que complementan la formación profesional del agente de desarrollo social: espíritu de iniciativa,
} 
habilidades tecnológicas y redes de interacción que le permitan cumplir con las demandas sociales.

$\mathrm{Al}$ promover la creación de vínculos y el intercambio, el agente construye nuevas hipótesis de trabajo. Debe desarrollar primero una capacidad de leer adecuadamente los fenómenos sociales. Su capacidad para reconocer situaciones es comprendida así, no tanto en el sentido de elaborar diagnósticos (necesarios, por cierto) como de interpretarlos de manera creativa. Debe poseer sólidas referencias teóricas de fondo, relativas al significado de su trabajo en la sociedad, a fin de poder usarlas de modo flexible y creativo en la aplicación diaria. También deberá saber cómo se producen las relaciones sociales entre los diversos actores en un territorio, dónde se colocan esos elementos que en potencia constituyen el capital social, y debe activar recursos que están latentes en una comunidad.

Otra información relevante es que se trata de trabajadores que cuentan con una buena escolarización. Como se observa en el cuadro 2, desagregada por tipo de institución, y tomando como referencia el grado profesional completo (incluido grado de maestría o alguna carrera profesional terminada), en las instituciones de asistencia privada encontramos $35.9 \%$, en las asociaciones civiles $36.7 \%$ y en otras (que incluye fundaciones y otro tipo de organizaciones) $25.1 \%$. El nivel más alto de doctorado desciende, pero esto es común en todos los sectores porque México cuenta aún con niveles bajos de posgrado en general.

\section{Cuadro 2}

Nivel de estudios de los trabajadores remunerados actuales, según tipo de organización

\begin{tabular}{|c|c|c|c|c|c|c|c|c|}
\hline 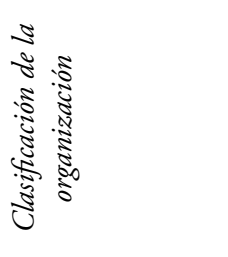 & 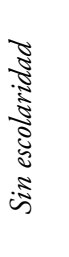 & 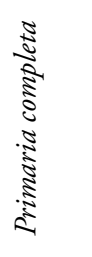 & 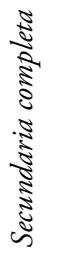 & 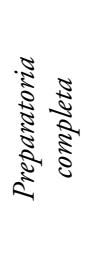 & 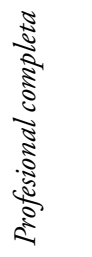 & 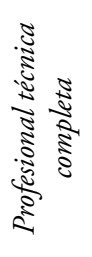 & 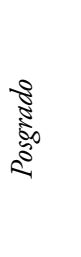 & 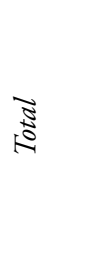 \\
\hline $\begin{array}{l}\text { Institución de } \\
\text { asistencia privada }\end{array}$ & 3.5 & 10.7 & 11.5 & 11.0 & 23.9 & 35.9 & 3.5 & 100.0 \\
\hline Asociación civil & 0.9 & 13.9 & 10.9 & 12.6 & 19.7 & 36.7 & 5.3 & 100.0 \\
\hline Otra & 0.3 & 10.5 & 2.6 & 27.6 & 33.9 & 25.1 & 0.0 & 100.0 \\
\hline
\end{tabular}

Fuente: Elaboración propia 2008. Proyecto Conacyt.

habilidad para concertar, capacidad para decidir y actuar en una lógica de equipo (team), lo que favorece los procesos de integración, cooperación y desarrollo de grupos y redes. 
El presupuesto de que las organizaciones abren nuevos nichos ocupacionales con profesiones emergentes, sólo se puede interpretar sobre el supuesto de crear nuevos servicios porque los resultados todavía no muestran el nacimiento de profesiones emergentes. Éste es un desafío pendiente sobre el cual se debe trabajar, incluso desde la profesionalización del sector y el reconocimiento de una nueva identidad profesional.

En la investigación se identificó además la polivalencia de los servicios que reportan las osc inscritas al Cluni (3,536 organizaciones). Se destaca de manera genérica la promoción y fomento educativo, cultural, artístico,

\section{Cuadro 3 \\ Actividades seńaladas por la Ley Federal de Fomento (número de organizaciones que la realizan)}

\begin{tabular}{lr}
\hline Asistencia social & 1,559 \\
$\begin{array}{l}\text { Apoyo a la alimentación popular } \\
\text { Cívicas, enfocadas a promover la participación ciudadana en asuntos }\end{array}$ & 830 \\
de interés público & 786 \\
$\begin{array}{l}\text { Asistencia jurídica } \\
\text { Apoyo para el desarrollo de los pueblos y comunidades indígenas }\end{array}$ & 1,127 \\
$\begin{array}{l}\text { Promoción de la equidad de género } \\
\text { Aportación de servicios para la atención a grupos sociales con capa- }\end{array}$ & 1,241 \\
$\begin{array}{l}\text { Cidades diferentes } \\
\text { Cooperación para el desarrollo comunitario }\end{array}$ & 974 \\
$\begin{array}{l}\text { Apoyo en la defensa y promoción de los derechos humanos } \\
\text { Promoción del deporte }\end{array}$ & 1,741 \\
$\begin{array}{l}\text { Promoción y aportación de servicios para la atención de la salud y } \\
\text { cuestiones sanitarias }\end{array}$ & 1,306 \\
$\begin{array}{l}\text { Apoyo en el aprovechamiento de los recursos naturales, la protección } \\
\text { del ambiente, la flora y la fauna, la preservación y restauración del } \\
\text { equilibrio ecológico, así como la promoción del equilibrio ecológico, }\end{array}$ & 1,089 \\
$\begin{array}{l}\text { la promoción del desarrollo sustentable a escala regional y comunita- } \\
\text { ria, de las zonas urbanas y rurales }\end{array}$ & \\
$\begin{array}{l}\text { Promoción y fomento educativo, cultural, artístico, científico y tec- } \\
\text { nológico }\end{array}$ & 2,132 \\
$\begin{array}{l}\text { Fomento de acciones para mejorar la economía popular } \\
\text { Participación en acciones de protección civil }\end{array}$ & 1,244 \\
$\begin{array}{l}\text { Prestación de servicios } \\
\text { Las que determinen otras leyes }\end{array}$ & 360 \\
\hline & 659 \\
\hline
\end{tabular}

Fuente: Indesol (2006), <http://www.corresponsabilidad.gob.mx>. 
científico y tecnológico brindado por 2,132 organizaciones; la cooperación para el desarrollo comunitario realizado por 1,741 organizaciones; seguida de la asistencia social que brindan 1,559 organizaciones; la promoción y aportación de servicios para atención de salud y cuestiones sanitarias donde participan 1,306 organizaciones para atender esta problemática. Las organizaciones inscritas al Cluni son en su mayoría prestadoras de servicios, si bien aún se necesita contar con datos más desagregados que den cuenta de la especificidad de estos servicios (cuadro 3).

\subsection{Los soportes financieros que limitan o incentivan distintas modalidades del empleo}

El sector sin fines de lucro se distingue de otros, como el público estatal y el privado, principalmente porque recibe fondos ya sea privados, de la cooperación internacional o del mismo gobierno, destinados a desarrollar sus tareas y objetivos. Cualquiera de estas organizaciones (fundaciones, instituciones de asistencia privada y las osc en general) se financian con aportaciones de sus asociados, donativos, legados, subsidios, venta de productos y servicios que se suman a los anteriores. Una condición del sector es que las instituciones no pueden redistribuir beneficios entre sus integrantes, restricción derivada de su carácter no especulativo. Esta característica (sin par en la empresa privada ni en la administración pública) permite que estas organizaciones puedan -al menos en teoría- disponer de recursos ilimitados, siempre que estén en condiciones de saber y poder atraer suficientes donaciones o trabajo voluntario (Fiorentini, 1997).

Como se observa en el cuadro 4 , las organizaciones analizadas reportan ingresos procedentes de las cuotas de sus socios en 31\%; por importancia, 26\% proviene del presupuesto que otorga el gobierno federal, estatal o municipal, y es significativo también el $20 \%$ que proviene de las donaciones de particulares. Cabe subrayar que una buena parte del financiamiento recabado se destina al pago de nóminas, integradas por administrativos o profesionales.

Como generadoras de empleo, las potencialidades de las osc están, en consecuencia, muy ligadas con la suficiencia de recursos financieros. Se puede constatar que mientras la dinámica de expansión de las instituciones en los últimos años constituye un activo favorable a la generación de empleo, los recursos financieros con que cuentan reducen su potencialidad, pues en muchas ocasiones no son continuos y en otras escasean o pueden incluso ser la razón del cierre de la misma institución. Así, enfrentan un problema serio para poder contar con recursos humanos suficientes y calificados, sometiéndolos a una inestabilidad que puede ser además la 


\begin{tabular}{|c|c|}
\hline $\begin{array}{c}\text { Cuadro } 4 \\
\text { Fuentes de ingresos }\end{array}$ & \\
\hline Fuente & $\begin{array}{r}2007 \\
(\%)\end{array}$ \\
\hline Cuotas de socios & 31 \\
\hline Donaciones de particulares & 20 \\
\hline Prestación de servicios & 12 \\
\hline Venta de productos & 6 \\
\hline Ingresos de la administración pública (local, estatal o federal) & 26 \\
\hline Financiamiento internacional & 3 \\
\hline Otras & 3 \\
\hline Total & 100 \\
\hline
\end{tabular}

Fuente: Elaboración propia 2008. Proyecto Conacyt.

causa que determina el tipo de contratos y el manejo de plazos limitados en la oferta de empleo.

\section{Reflexiones finales}

A lo largo de este estudio abordamos algunos elementos para reflexionar en torno a las potencialidades de trabajo y empleo que se generan por la prestación de servicios de proximidad y/o relacionales brindados por el sector sin fines de lucro en México. Consideramos que la adscripción de los trabajadores a las osc, como voluntarios, donadores o remunerados, constituye un activo que sustenta los poderes potenciales de las instituciones. Las peculiaridades del tipo de trabajadores que colaboran con estas organizaciones determinan la percepción de los recursos humanos como activos sociales, como recursos y/o bienes que no necesariamente están del todo motivados por razones monetarias. Esta singularidad afecta las relaciones laborales ya que los voluntarios pueden trabajar sin goce de sueldo, o apenas si reciben pagos compensatorios; los asalariados devengan pagos menores que los que alcanzarían por la prestación de los mismos servicios en una organización lucrativa. Suelen desempeñarse sin prestaciones ni contratos estables, en función del compromiso personal con la labor que realiza la institución o de colaboración con las personas que se ven beneficiadas por esos servicios.

Con base en los datos obtenidos, podemos advertir que existe una relación entre la expansión de las osc como nichos ocupacionales ante los cambios en el Estado social tradicional, y la descentralización de sus servicios, los cambios en el modo de vida de las personas y su manifestación de nuevas 
necesidades no cubiertas por ese mismo Estado de bienestar. En el marco de los cambios, como parte de un proceso más general de reestructuración estatal, estas organizaciones junto a otra pluralidad de actores entre los que se encuentran los diversos niveles de gobierno, fundaciones nacionales e internacionales, organizaciones empresariales, religiosas, de asistencia, de desarrollo, el mismo sector privado, la universidad, entre otros, participan a través de una multiplicidad de acciones y modalidades en la prestación de servicios sociales. No obstante, las asociaciones que desarrollan regularmente actividades vinculadas con la prestación de servicios, en el marco de las políticas públicas, son un subgrupo (de tamaño variable) del total de organizaciones que componen el heterogéneo universo de este sector.

Este preciso abanico de organizaciones desarrolla una serie de acciones y actividades cuyas intervenciones se llevan adelante en nombre del interés público, la movilización, la participación en espacios de consulta para el diseño de políticas públicas y la colaboración en la implementación y en la evaluación de las mismas.

La convivencia dentro de las osc entre trabajadores voluntarios y remunerados, la organización de trabajos flexibles no siempre asociados a las formas tradicionales del empleo asalariado, sus modalidades como trabajos por cuenta propia, por honorarios, por tiempo determinado, trabajo provisional, entre otros esquemas de contratación, constituyen condiciones favorables para la creación de ocupación, pero por otra parte, demuestran todavía una cierta precariedad en cuanto al factor de perdurabilidad del empleo, y de vulnerabilidad por falta de prestaciones sociales.

Las categorías analíticas que hemos elegido como marco teórico resultan adecuadas. La economía solidaria y los servicios de proximidad y/o relacionales (si se considera el sector de la economía en donde se insertan y el tipo de servicios que brindan) respondieron a las categorías enunciadas y nos permitieron estudiar las características correspondientes de empleo.

En este sentido, el presupuesto de que en estas organizaciones se abren nuevos nichos ocupacionales con profesiones emergentes, sólo se puede interpretar en la inteligencia de los nuevos servicios que atienden. Servicios intangibles con un fuerte contenido relacional que exigen de los empleados conocimientos, profesionalización y una identidad definida específicamente para ese sector. Sin embargo, al carecer de una adecuada reflexión en torno a sus características específicas, los confunde con otros trabajadores del sector privado o de gobierno, restándoles sus peculiaridades al homologarlos a todos de manera genérica.

Pero no es así. Se trata de un sector calificado. La mayoría de sus trabajadores cuenta con estudios de secundaria, preparatoria y, sobre todo, profesionales egresados de carreras universitarias. 
Las organizaciones estudiadas están reguladas por otros principios ordenadores asociados con la cohesión social, principios como la solidaridad, la relacionalidad y reciprocidad más que por principios de la racionalidad técnico-instrumental, aunque esta regulación aún tiene que sistematizarse para que el sector se la pueda apropiar.

Hay muchos desafíos pendientes por afrontar cuando de actividad, empleo y trabajo se habla. No sabemos con certeza cuánto empleo genera este sector. Si bien las osc han crecido y se han desarrollado significativamente en los últimos años, sabemos que incorporan muchos recursos humanos a sus organizaciones, pero por la falta de datos estadísticos, por la carencia de normas específicas, por la falta de respuesta sobre estos indicadores por parte de las mismas organizaciones, se vuelve imposible su cuantificación. Sin lugar a dudas, se trata de un sector -sobre todo en México-invisible o poco reconocido en cuanto a su autonomía, sistematizado desde sus propias características. La disponibilidad financiera es el factor principal en la decisión de incorporar a más trabajadores renumerados; hay que reconocer que la condición de los mismos hasta ahora es precaria debido a las condiciones de desventaja ya descritas (remuneraciones bajas y ninguna prestación social). La continuidad de su trabajo depende muchas veces de los proyectos y del financiamiento con que cuentan las organizaciones.

Se requiere de políticas públicas que además de promocionar el sector, se ocupen de hacerlo visible y potenciarlo en su justa dimensión: un segmento que trabaja en pos de la inclusión y la cohesión sociales. Un mayor conocimiento del sector permitirá una mejor regulación y la configuración de un estatuto para estas nuevas figuras profesionales.

\section{Bibliografía}

Ackeman, Susan Rose (1996), "Altruism nonprofits, and economic theory", Journal of Economic Literature, 34 (2), American Economic Association, Pittsburgh, pp. 701-728.

Bresser Pereira, Luiz Carlos y Nuria Cunill Grau (1998), Lo público no estatal en la reforma del Estado, CLAD, Caracas.

Cabin, Philippe (1999), Les organizations: état des savoirs, Sciences Humaines Éditions, París.

Caselli, Clara (1998), "Il non profit: aspetti aziendali", Persone e imprese, 8 (2), Editore Liguori, Nápoles, pp. 68-79. 
Comisión Europea (1993), Europa en un mundo cambiante. Relaciones exteriores de la Comununidad Europea, Comisión Europea, Luxemburgo.

Clotfeler, Charles (1992), Who benefits from the nonprofit sector?, University Chicago Press, Chicago.

Donatti, Pierpaolo (1996), Sociologia del terzo settore, La Nuova Italia Scientifica, Roma.

Eme, Bernard (1993), Lecture d'Habermas et éléments provisoires d'une problématique du social solidariste d'intervention, LSCI, Iresco-CNRS, París.

Fiorentini, Gorgio (1997), Organizzazione non profit e di voluntariato, Etas Libri, Milán.

Girardo, Cristina (2003), La formación para el trabajo de los jóvenes (desafios y estrategias) desde las organizaciones de la sociedad civil, Universidad Nacional Autónoma de México, México.

Girardo, Cristina (2005), La formación de agentes de desarrollo social, México, investigación financiada por Indesol en los programas de Coinversión-México. Informe final entregado al Indesol, Mimeo.

Girardo, Cristina (2007), "Profesionalización y desarrollo organizacional en las osc", en Cristina Girardo (coord.), La profesionalización de las OSC en México. Actores y estrategias, El Colegio Mexiquense, Zinacantepec, pp. 17-46.

Guadarrama, Gloria y Cristina Girardo (2008), "Empleo en organizaciones de la sociedad civil: una aproximación a las instituciones de asistencia privada en el Estado de México", El Colegio Mexiquense, Zinacantepec, documento de investigación núm. 126.

Laville, Jean Louis (2004), Economia socialy solidaria. Una visión europea, Altamara, Buenos Aires.

Mochi, Prudencio (2003), Usos y apropiación de las TIC'S por parte de las OSC, México, investigación financiada por Indesol en los programas de Coinversión-México. Informe final entregado a Indesol, mimeo. 
Neffa, Julio (2005), Actividad, empleo y desempleo: conceptos y definiciones, Miño y Dávila, Buenos Aires.

Olvera, Alberto (coord.) (1999), La sociedad civil: de la teoría a la realidad, El Colegio de México, México.

Salomon, Lester y Helmut Anheimer (1997), Defining the nonprofit sector. A cross-national analysis, Institute for Policy Studies-The Johns Hopkins University-Manchester University Press, Manchester.

Salomon, Lester y Helmut Anheimer (1998), The non-profit sector in the developing word, Manchester University Press, Manchester.

Selznick, Philippe (1996), "Institutionalism old and new”, Administrative Science Quarterly, 41, Cornell University, Nueva York, pp. 270-277.

Weisbrod, Burton Allen (1991), The nonprofit economy, Harvard University Press, Watertown.

Recibido: 30 de junio de 2009. Reenviado: 5 de mayo de 2011. Reenviado: 17 de junio de 2011. Aceptado: 18 de julio de 2011.

Cristina Girardo. Es doctora en estudios latinoamericanos por la Universidad Nacional Autónoma de México (UNAM), asimismo realizó un doctorado en pedagogía en la Universidad de Torino, en Italia. Actualmente es investigador nivel I en el Sistema Nacional de Investigadores (SNI) y es profesora-investigadora en El Colegio Mexiquense. Su línea de investigación actual es el empleo en las organizaciones de la sociedad civil. Entre sus publicaciones destacan: Cuaderno de investigación: una aproximación cualitativa sobre el empleo y sus modalidades en las organizaciones de la sociedad civil en México, El Colegio Mexiquense, Zinacantepec (2009); Trabajo voluntario y trabajo informal en el sector solidario. El empleo en las instituciones mexiquenses de asistencia privada, El Colegio Mexiquense, Zinacantepec (2009); "La construcción, apropiación y difusión del conocimiento en la profesionalización de agentes para el desarrollo local", en Jorge Basave Kunhardt y Miguel Ángel Rivera Ríos (coords.), Globalización, conocimiento y desarrollo, t. II, UnAm, México, pp. 101-124 (2009). 
Prudencio Mochi. Es doctor en estudios latinoamericanos por la Universidad Nacional Autónoma de México (UNAM), asimismo realizó un doctorado en ciencias políticas en la Universidad de Torino, en Italia. Actualmente es investigador nivel I en el Sistema Nacional de Investigadores (SNI) y es profesor-investigador en el Centro Regional de Investigaciones Multidisciplinarias (CRIM) de la UNAM. Su línea de investigación actual es: uso y apropiación de Tic por parte de las osc, el desarrollo local y los clusters tecnológicos. Entre sus publicaciones destacan: "La industria del software: un sector emergente en América Latina", en Alejandro Dabat Latrubesse y José de Jesús Rodríguez Vargas (coords.), Globalización, conocimiento y desarrollo, t. I, UnAM, México, pp. 101-124 (2009); "Aportes, temas y enfoques planteados desde las ciencias sociales para abordar el desarrollo local y la territorialización de políticas públicas”, en Cristina Girardo (coord.), El desarrollo local en México: aportes teóricos y empiricos para el debate, UADY, Mérida, pp. 33-68 (2008); "La industria del software en México y en América Latina", en Miguel Ángel Rivera Ríos y Alejandro Dabat (coords.), Cambio histórico mundial, conocimiento y desarrollo, unam-Juan Pablos, México, pp. 303-325 (2007). 